Published in final edited form as:

Adv Exp Med Biol. 2014 ; 844: 85-98. doi:10.1007/978-1-4939-2095-2_5.

\title{
Systems Biology of Platelet-Vessel Wall Interactions
}

\author{
Yolande Chen, \\ RH Lurie Comprehensive Cancer Center, Northwestern University Feinberg School of Medicine, \\ Rm 5-220, 303 E. Superior St., Chicago, IL 60611, USA

\section{Seth Joel Corey,} \\ Department of Pediatrics and Cell \& Molecular Biology, Northwestern University Feinberg School \\ of Medicine and Lurie Children's Hospital of Chicago, Chicago, IL, USA \\ Oleg V. Kim, and \\ Department of Applied and Computational Mathematics and Statistics, University of Notre Dame, \\ Notre Dame, IN, USA

\section{Mark S. Alber} \\ Department of Applied and Computational Mathematics and Statistics, University of Notre Dame, \\ Notre Dame, IN, USA; Department of Medicine, Indiana University School of Medicine, \\ Indianapolis, IN, USA
}

\section{Abstract \\ Platelets are small, anucleated cells that participate in primary hemostasis by forming a hemostatic plug at the site of a blood vessel's breach, preventing blood loss. However, hemostatic events can lead to excessive thrombosis, resulting in life-threatening strokes, emboli, or infarction. \\ Development of multi-scale models coupling processes at several scales and running predictive model simulations on powerful computer clusters can help interdisciplinary groups of researchers to suggest and test new patient-specific treatment strategies.}

\section{Keywords}

Platelets; Blood vessels; Hemostasis; Thrombosis

\section{Multiple Functions of Platelets in Blood Clot Development}

First observed through simple microscopy in the nineteenth century, platelets and leukocytes interact with blood vessel walls [1,2]. Since those first observations, an impressive body of information has accumulated to establish the centrality of platelets in vascular thrombosis. The main steps in thrombosis formation classically include the tethering, rolling/ translocation, and adhesion of leukocytes and platelets to the exposed matrix at the damaged blood vessel site. Arterial thrombosis remains the most common cause of myocardial infarction and stroke, resulting in significant morbidity and mortality. To prevent thrombosis, many patients are treated with antiplatelet agents [3]. By producing chemical messengers, 
microparticles, and vascular changes, platelets also promote cancer and inflammation [4-6]. Therefore, there is a great need to understand more platelet-leukocyte-endothelial interactions (Fig. 5.1) and translate that into more effective, less toxic therapies.

A major challenge in quantitative understanding of hemostasis/thrombosis is to integrate various processes occurring during thrombus development. To predict how variations of multiple factors associated with platelet activity affect thrombus development is of great biomedical importance. However, there are significant challenges in developing such understandings. For instance, platelet-vessel wall receptor-ligand interactions occur at nanometer scale, whereas blood flow dynamics in the vicinity of a thrombus is a macroscopic event developing over the scale of hundreds of micrometers to millimeters. Coupling various processes is a complex, challenging task.

By reviewing main platelet functions, their roles in hemostasis/thrombosis, and computational approaches to simulate clotting events, this chapter establishes a rationale for a systems approach to platelet physiology. These include modeling of coagulation reactions, platelet activation, platelet dynamics, platelet-platelet interactions, and blood flow. The integrative modeling approaches are described to provide the basis for multi-scale computational models of thrombus development. First, biological background on platelet functioning is given, including platelet adherence and activation, intracellular and extracellular signaling, relation to tumor metastasis, and global approaches to study plateletvessel wall interactions. Then, several recent integrative modeling methods of thrombus development involving cellular signaling, platelet-platelet, platelet-flow, and platelet-wall interactions are highlighted. Finally, a concluding perspective is offered on the role of platelets in hemostasis/thrombosis and tumor progression as well as the role of system biology in testing new therapeutic targets.

\section{Platelet Adherence and Activation}

Platelets interact with their environment through specialized receptors, many of which are integrins [7]. One principle mediator is platelet P-selectin (CD62P), a cell adhesion molecule stored in platelet alpha granules that interacts with P-selectin ligand 1 (PSGL-1, or CD162, found on leukocytes and endothelial cells). Endothelial cells possess granules called Weibel-Palade bodies, which release von Willebrand Factor (vWF) and P-selectin when activated. Interaction between P-selectin and its ligand occurs during tethering and rolling on the endothelium [1]. Importantly, the platelet surface receptor for vWF is glycoprotein Ib (GPIb; CD42). Lack of cleavage of high molecular weight vWF multimers results in thrombotic thrombocytopenic purpura, a catastrophic disorder. On the other hand, defective GPIb expression or activity results in excessive bleeding, the Bernard-Soulier disorder. vWF binding to GPIb induces downstream cytoskeletal actin rearrangement via FilaminA. FilaminA which regulates intracellular signaling [8] is responsible for a solid anchorage of GPIb [9]. Filamin A mutations have recently been found as a cause for thrombocytopenia [10] and abnormal platelet function [11].

Another critical mediator for platelet interactions is integrin aIIb $\beta 3$ (CD41), a cell surface receptor for fibrinogen. This integrin is defective in individuals suffering from Glanzmann's 
thrombasthenia, a bleeding disorder. When fibrinogen binds to the integrin, a cascade of downstream events, which include cytoskeletal rearrangements via talin and other signaling involving rous sarcoma (SRC) and focal adhesion kinase (FAK)-tyrosine kinases [12], is set in motion. Equally important is the presence of collagen receptors at the surface of the platelet, the better known of which are GPVI and $a 2 \beta 1$. GPVI in particular seems to have a prominent role during collagen exposure to platelet surface and subsequent activation [12]. Platelets also express the C-type lectin receptor (CLEC-2, which triggers a downstream signaling cascade similar to that of GPVI, including activation of the spleen tyrosine kinase (Syk)) [12].

G-protein-coupled receptors include the thrombin receptor, adenosine diphosphate (ADP) receptors (P2Y1, P2Y12 which is a target for several "antiplatelet" drugs in thrombotic disease treatment) $[3,13]$, and thromboxane $\mathrm{A} 2$ receptor. They are activated in response to soluble factors from the blood. Exposure of the subendothelial matrix provides additional potent activators of platelet activation and coagulation. Platelet receptors recognize matrix components, collagen (via a $2 \beta 1, \mathrm{GPVI}$ ), and laminin (via a $6 \beta 1$ ). Targeting of the laminin receptor, like the integrin $\alpha 6 \beta 1$, has been shown to be a promising strategy in the treatment of arterial thrombosis [14]. Recently, platelets were found to express CXCR6, the receptor specific to chemokine CXCL16. CXCL16 present on atherosclerotic lesions was found to enhance platelet adhesion to the endothelium after high arterial shear stress and to injured vascular wall [15]. Other cell surface proteins on platelets, such as Eph kinases and EphrinB1 [16], semaphorins [17] or Gas6 receptors [18], may promote thrombus formation. Gap junction channels, such as connexin 37 [19], may also enhance thrombus formation.

The list of platelet cell surface receptors or adhesion molecules will likely continue to grow, adding to complexity of platelet interactions and function. For instance, by expressing CD40-L, Fc receptors to immunoglobulins, and toll-like receptors [5], platelets serve as effectors in the immune system. Platelet-derived microparticles containing pro-inflammatory cytokines such as IL-1 $\beta$ contribute to inflammation [6].

\section{Intracellular Cell Signaling and Inter-platelet Signaling}

Resulting from those diverse ligand-receptor stimuli (Fig. 5.2), downstream signaling events involve phospholipid metabolism, generation of cAMP and cGMP second messengers, and $\mathrm{Ca}^{2+}$ release from the dense tubular system. These pathways lead to cytoskeletal reorganization, calpain activation, and signal amplification and diversification due to protein and lipid kinases. Phosphatase activity increases too [20]. Many of these pathways intersect or interact with each other in a complex fashion. Besides outside-inside signaling, there is also inside-outside signaling, as in the case of aIIb $\beta 3$ integrin activation [12, 21].

\section{Extraplatelet Signaling}

Upon platelet stimulation, alpha or dense granule release occurs, leading to platelet activation amplification, since the released substances (fibrinogen, vWF,ADP, thromboxane A2, thrombospondin-1) will further activate platelets and contribute to hemostasis. One adaptive measure is to induce blood vessel constriction, which is achieved through 
thromboxane A2. However, alpha granules release not only prothrombotic but also proangiogenic (VEGF) and anti-angiogenic (endostatin, thrombospondin) factors [22-25]. Intensively studied are platelet microparticles and exosomes. Microparticles are shed from membranes and their size varies from 100 to $1000 \mathrm{~nm}$ [26], while exosomes are secreted and overall smaller (30-100 nm) [27]. Microparticles are shed from the platelet membrane and carry tissue factor activity, thus being procoagulant $[12,28]$. Exosome secretion from platelets has also been described, but their role is less well known [29]. Inflammation contributes to thrombus formation through the interaction of platelets with leukocytes (neutrophils and monocytes) [30, 31]. Indeed, thrombosis could more generally be seen as an effector of innate immunity [32]. Moreover, neutrophils also contribute to thrombus formation, including via formation of neutrophil extracellular traps (NET) [33-37].

When a blood vessel is injured, the subendothelial matrix is exposed, and matrix components such as the collagens or laminins serve as potent activators of platelet activation. Furthermore, endothelial cells release prothrombotic factors such as vWF from their Weibel-Palade bodies. Interestingly, vWF release from the endothelial Weibel-Palade bodies is dependent on essential autophagy genes Atg5 or Atg7, and pharmacological inhibitors of autophagic flux lead to increased bleeding time [38]. On the other hand, the endothelium also releases substances that are inhibitory to platelet activation, by secreting nitric oxide (NO) and the downstream modulation of cGMP levels [39-43], or by secreting prostacyclin(PGI2) [44].

On a different scale, platelet contraction forces depend on microenvironment stiffness [45] and platelet adhesion and spreading depends on local microenvironment geometry [46]. Stresses developed by contracting platelets significantly alter thrombus internal structure and were recently shown to strongly deform embedded erythrocytes [47]. Vessels can be made susceptible to injury depending on vessel geometry, biophysical and rheological forces from the blood flow that will produce turbulence and shear. Models are being developed, where the role of vWF is still being found to be crucial [48]. Progression of an injured endothelium, resulting from a combination of flow shear, inflammatory state, and dyslipidemia, results in atherosclerosis and atheroma plaques, which by themselves lead to specific atheroma-platelet interactions [15, 44].

\section{Platelets and Tumor Metastasis Through the Vessel Wall}

Apart from their role in thrombosis and inflammation/immunity, platelets are also currently under intense scrutiny for their role in cancer metastasis dissemination. Indeed, a study has found that interactions between platelets and tumor cells induce an invasive mesenchymallike phenotype and enhance in vivo metastasis. This effect is mediated by platelet-derived TGF $\beta$, which activates the Smad and NF- $\mathrm{kB}$ pathways in cancer cells [4]. Specific to vessel wall effects, tumor cell-activated platelets secreted ADP, which facilitates cancer cell penetration past blood vessels. The P2Y2 receptor on blood vessel cells is necessary for this effect [49]. 


\section{Global Approaches in Studying Platelet-Vessel Wall Interactions}

To discover molecular mechanisms of platelet interactions with the vessel wall, researchers have been relying on narrowed and focused approaches (Fig. 5.3). One example is observing human disease and trying to pin down the molecular defect. Then one might want to search for partners of known actors. Subsequently, to confirm the relevance, one might pursue on knocking down or knocking out an element and observing the cell or organism for modified behaviors. More recently, researchers have been adding new global approaches in the field of biology, enriching knowledge through global and systemic approaches [50]. These new global approaches include:

1. Mutagenesis screening by N-ethyl-N-nitrosourea (ENU). For example, BcL-xL was discovered to regulate platelet half-life or platelet number [51].

2. Genome-wide association studies (GWAS). Human genomic variations are associated with cardiovascular outcomes, platelet size, or number, as reported by European study consortiums [52]. Jones et al. found that single nucleotide variants in platelet endothelial aggregation receptor 1 (PEAR1), guanine nucleotide exchange factors (GEFs) for Rho family GTPases (VAV3), and IP3 receptor (ITPR1) were associated with modified platelet response to platelet agonists [53].

3. Transcriptomics. Rowley et al. recently reported a comprehensive transcriptome study of human and mouse platelets [54]. This approach led to the discovery of connexin 37 in platelet aggregation [19].

4. Proteomics. Comprehensive platelet proteomics have been performed [55] and can be further narrowed down to subfields as "secretome" or "phosphoproteome" [56]. These proteomic studies revealed that secretogranin III, cyclophilin A, and calumenin were secreted by platelets after thrombin stimulation and found in atherosclerotic plaques [57] and nitrous oxide-treatment abrogated platelet activation by thrombin and prevented thrombin-induced translocation of gelsolin, filamin, 14-3-3 $\zeta$, phosphatidylinositol 3-kinase-gamma isoform, and growth factor receptor-bound protein 2 (Grb2) [58].

5. Network analysis. Network analysis tools are being developed and databases are being made available online. For example, cPlateletWeb (http:// plateletweb.bioapps.biozentrum.uni-wuerzburg.de) is an Internet-based platform organizing signaling network $[59,60]$. A study based on PlateletWeb found a novel interaction between vasodilator-stimulated phosphoprotein and Abelson interactor 1 in human platelets [61]. Another database is Reactome (http:// www.reactome.org), where extensive data have been collected, analyzed, and grouped in different pathways [62].

6. Informatics for modeling and simulations. Bioinformatics can be used to develop models for simulating platelet function, thrombus formation associated with proor anticoagulant gradients, and different flow conditions [63-69]. Indeed, thrombus formation studies have mostly focused on separate components of thrombogenesis, which can be numerous and subgrouped into categories: 
coagulation cascades of blood coagulation factors, platelet adhesion to the vascular wall, platelet aggregation among themselves, internal platelet activation phenomena, platelet substance release and amplification reaction, white blood cells'roles in thrombus formation, vessel wall product release, vessel wall injury and exposure of thrombogenic elements, atheroma genesis, and blood flow shear variation and impact.

\section{Integrative Multi-scale Modeling Approaches}

Integrating the relative role of each of the mentioned elements to model thrombus formation has been challenging. Various modeling approaches have been proposed, that integrate a certain number of processes or scales, and tested with the assistance of simulations implemented on large computer clusters (see $[63,65,70,83]$ for review). One target goal for future research would be to improve modeling ability to predict platelet/vessel wall behavior and thrombus formation by integrating simulations of the molecular signature characteristics, mechanical properties of agonist/antagonist, blood flow and viscoelastic properties of a blood vessel. Below we outline several existing modeling approaches that combine several scales.

Explicit incorporation of single platelet dynamics into a three-dimensional thrombus formation model has been described in Pivkin et al. [71] where each platelet was represented in a simplified way as a spherical object, while red blood cells were treated using continuum submodel describing their density. The model also included an ADP-induced platelet activation mechanism. Model simulations accurately reproduced the thrombus growth rate as a function of blood velocity obtained in experiments [72].

Fogelson and Guy developed a microscale platelet aggregation model in which individual platelets were modeled as fluid-filled closed membranes immersed in a viscous liquid [73]. This model allowed for simulations of individual platelet motion and their interactions with each other and with surrounding medium. In the model by Mody and King [74], the hydrodynamic effects of the oblate spheroidal shape of platelets and the proximity of a wall on cell-cell collisions were investigated. Collision time and contact area and collision frequency were compared between spherical shape vs. platelet-type oblate shape on one hand and presence or not of a proximal bounding wall. The approach used calculation of forces and torques acting on each particle in the fluid system (gravity, bond forces, and repulsion between two surfaces in close proximity). The study showed that the contact time between two platelets during collision close to the wall was greater than the contact time during a collision far from the wall. The wall proximity had a greater influence on plateletplatelet collisions than on sphere-sphere collisions. The method also used Huang and Hellums' mathematical model of the shear-induced platelet aggregation [75-77], where high shear resulted in increased platelet aggregation.

Leiderman and Fogelson [78] described a model of blood coagulation under flow that included coagulation biochemistry, chemical activation, and deposition of platelets, and a two-way interaction between fluid dynamics and growth of platelet mass. Expanding on a previously published Kuharsky and Fogelson model [79], this approach now described how 
tissue factor threshold triggered production of thrombin and how the wall shear rate and near-wall increased platelet concentration affected thrombus growth. The porous nature of the thrombus, allowing for advective and diffusive transport within itself, was also accounted for in the extended model. Xu et al. [63, 80-83] proposed a multi-scale model-coupling submodels of coagulation reactions, platelet dynamics, and blood flow, where platelets were represented as extended objects with fluctuating boundaries based on the cellular Potts model [84].

The importance of quantifying transport of coagulation factors within thrombus microenvironment was emphasized in Ref. [69]. By combining in vitro experiments and continuum-modeling approach of thrombus hydrodynamics, authors showed that both diffusivity and advection of blood proteins through the porous thrombus structure affect platelet-thrombus interaction and play an essential role in blood clot growth dynamics. Following this work, Stalker et al. demonstrated, using mouse injury model, that regional platelet-packing density emerged in parallel with differences in intrathrombus molecular transport and predicted that these differences affect thrombus growth and stability [85].

Wu et al. [86] presented a three-dimensional multi-scale platelet-blood flow-vessel wall interaction model, which combined three biological scales crucial for the early platelet aggregation. The model included hybrid cell membrane submodel of platelet elasticity, stochastic receptor-ligand binding submodel of cell adhesion kinetics and Lattice Boltzmann submodel of blood flow. Adhesion kinetics involved specific receptor-ligand pairs, namely vWF-GpIb complexes. At subcellular level, to simulate vWF-GpIb and GpIbvWF-GpIbabinding, individual molecules were represented by elastic springs. This was justified by the demonstration that the receptor-ligand binding is probabilistic in nature. Individual filaments of the cytoskeleton network of platelet membrane were modeled as coarse-grained harmonic potentials. At cellular level, a novel continuum description of the cell membrane was used. The subcellular and cellular components were integrated by distributing GpIba receptors over verticies of the cytoskeleton network and by superimposing the lipid bilayer and the network. The model allowed investigation of how platelet stiffness, GPIb receptor expression, and platelet-platelet interaction affect plateletwall adhesion quantified in terms of platelet pause time. To reduce the computational time cost, the model was implemented on graphical processing units (GPUs) computer cluster. Predictive simulations revealed that platelet deformation, interactions between platelets in the vicinity of the vessel wall, as well as the number of functional GPIba platelet receptors played significant roles in the platelet adhesion to the injury site (Fig. 5.4, from Ref. [86]).

Recently, a multi-scale model was presented in Flamm, Diamond et al. [64, 65, 87], which included four components: the fluid flow (using a lattice Boltzmann method), the transport of soluble substances (using convection-diffusion-reaction equations), motion and binding of platelets leading to their deposition (using a lattice kinetic Monte Carlo algorithm), and the activation state of each platelet (using a neural network for cellular signaling). A pairwise agonist scanning approach had been found to allow handling of large datasets of measured calcium mobilization to predict an individual's platelet responses to pairwise combinations of ADP (which activates P2Y1/P2Y12 receptors), U46619 (which has properties similar to Thromboxane A2), and convulxin (which activates GPVI receptor to 
collagen). A neural network for cellular signaling was used to predict patient-specific responses to drugs. The resulting simulations were compared with experimental results in a system using blood flowing on collagen in microfluidic devices at different shear rates. The simulations were used to predict the individual's drug sensitivity to cyclooxygenase (COX) inhibitors and P2Y1 receptor antagonists in three different blood donors.

\section{Perspective and Conclusions}

Increasing and organizing our knowledge on platelet-vessel wall interactions and combining it with novel multi-scale computational models to test new biological hypothesis will help devise treatments for human disease where excessive thrombosis occurs, while attempting to minimize the risk of bleeding. Moreover, platelets and vessels are not only implicated in "pure" thrombotic states and inflammatory disease but also in other pathological processes such as cancer and metastases. Furthermore, how both platelets and blood vessels may contribute to tumor growth and tumor dissemination can be by itself a part of a systems approach to cancer biology. Relying only on in vivo studies is impractical and time consuming. Genetic knockout animal models have their own limits, as nonhuman animals' biology differs from human biology in many aspects, and a complete functional knockout might not yield information on certain conditions, where a dosage effect or a mutated state might be the actual determinant pathogenesis. While hypothesis-based experiments are being performed, concomitantly developing and refining multi-scale models and running simulations on powerful computer clusters will enable biomedical community to accelerate testing of new therapeutic targets. Systems biology is thus becoming a novel empowering tool to devise new less toxic treatments more efficiently and economically.

\section{Acknowledgments}

Research of Mark Alber and Oleg Kim reported in this publication was supported by NIH U01HL116330, Yolande Chen by an American Heart Association Post-Doctoral Fellowship, and Seth Corey by NIH R21HL106462.

\section{References}

1. Wagner DD, Frenette PS. The vessel wall and its interactions. Blood. 2008; 111(11):5271-81. [PubMed: 18502843]

2. Jackson SP. Arterial thrombosis-insidious, unpredictable and deadly. Nat Med. 2011; 17(11):142336. [PubMed: 22064432]

3. Michelson AD. Antiplatelet therapies for the treatment of cardiovascular disease. Nat Rev Drug Discov. 2010; 9(2):154-69. [PubMed: 20118963]

4. Labelle M, Begum S, Hynes RO. Direct signaling between platelets and cancer cells induces an epithelial-mesenchymal-like transition and promotes metastasis. Cancer Cell. 2011; 20(5):576-90. [PubMed: 22094253]

5. Rondina MT, Weyrich AS, Zimmerman GA. Platelets as cellular effectors of inflammation in vascular diseases. Circ Res. 2013; 112(11):1506-19. [PubMed: 23704217]

6. Boilard E, et al. Platelets amplify inflammation in arthritis via collagen-dependent microparticle production. Science. 2010; 327(5965):580-3. [PubMed: 20110505]

7. Kauskot A, Hoylaerts MF. Platelet receptors. Handb Exp Pharmacol. 2012; 210:23-57.

8. Falet $\mathrm{H}$, et al. A novel interaction between FlnA and Syk regulates platelet ITAM-mediated receptor signaling and function. J Exp Med. 2010; 207(9):1967-79. [PubMed: 20713593] 
9. Feng S, et al. Filamin A binding to the cytoplasmic tail of glycoprotein Ibalpha regulates von Willebrand factor-induced platelet activation. Blood. 2003; 102(6):2122-9. [PubMed: 12791664]

10. Nurden $P$, et al. Thrombocytopenia resulting from mutations in filamin A can be expressed as an isolated syndrome. Blood. 2011; 118(22):5928-37. [PubMed: 21960593]

11. Berrou E, et al. Heterogeneity of platelet functional alterations in patients with filamin A mutations. Arterioscler Thromb Vasc Biol. 2013; 33(1):e11-8. [PubMed: 23117662]

12. Versteeg HH, et al. New fundamentals in hemostasis. Physiol Rev. 2013; 93(1):327-58. [PubMed: 23303912]

13. Gachet C. P2Y(12) receptors in platelets and other hematopoietic and non-hematopoietic cells. Purinergic Signal. 2012; 8(3):609-19. [PubMed: 22528678]

14. Schaff M, et al. Integrin alpha6beta1 is the main receptor for vascular laminins and plays a role in platelet adhesion, activation, and arterial thrombosis. Circulation. 2013; 128(5):541-52. [PubMed: 23797810]

15. Borst $\mathrm{O}$, et al. The inflammatory chemokine CXC motif ligand 16 triggers platelet activation and adhesion via CXC motif receptor 6-dependent phosphatidylinositide 3-kinase/Akt signaling. Circ Res. 2012; 111(10):1297-307. [PubMed: 22927331]

16. Prevost N, et al. Signaling by ephrinB1 and Eph kinases in platelets promotes Rap1 activation, platelet adhesion, and aggregation via effector pathways that do not require phosphorylation of ephrinB1. Blood. 2004; 103(4):1348-55. [PubMed: 14576067]

17. Zhu L, et al. Regulated surface expression and shedding support a dual role for semaphorin 4D in platelet responses to vascular injury. Proc Natl Acad Sci U S A. 2007; 104(5):1621-6. [PubMed: 17244710]

18. Angelillo-Scherrer A, et al. Role of Gas6 receptors in platelet signaling during thrombus stabilization and implications for antithrombotic therapy. J Clin Invest. 2005; 115(2):237-46. [PubMed: 15650770]

19. Vaiyapuri S, et al. Gap junctions and connexin hemichannels underpin hemostasis and thrombosis. Circulation. 2012; 125(20):2479-91. [PubMed: 22528526]

20. Senis YA. Protein-tyrosine phosphatases: a new frontier in platelet signal transduction. J Thromb Haemost. 2013; 11(10):1800-13. [PubMed: 24015866]

21. Stalker TJ, et al. Platelet signaling. Handb Exp Pharmacol. 2012; 210:59-85.

22. Italiano JE Jr, et al. Angiogenesis is regulated by a novel mechanism: pro- and antiangiogenic proteins are organized into separate platelet alpha granules and differentially released. Blood. 2008; 111(3):1227-33. [PubMed: 17962514]

23. Italiano JE Jr, Battinelli EM. Selective sorting of alpha-granule proteins. J Thromb Haemost. 2009; 7(Suppl 1):173-6. [PubMed: 19630794]

24. Battinelli EM, Markens BA, Italiano JE Jr. Release of angiogenesis regulatory proteins from platelet alpha granules: modulation of physiologic and pathologic angiogenesis. Blood. 2011; 118(5):1359-69. [PubMed: 21680800]

25. Battinelli EM, et al. Anticoagulation inhibits tumor cell-mediated release of platelet angiogenic proteins and diminishes platelet angiogenic response. Blood. 2014; 123(1):101-12. [PubMed: 24065244]

26. Thery C, Ostrowski M, Segura E. Membrane vesicles as conveyors of immune responses. Nat Rev Immunol. 2009; 9(8):581-93. [PubMed: 19498381]

27. Raposo G, Stoorvogel W. Extracellular vesicles: exosomes, microvesicles, and friends. J Cell Biol. 2013; 200(4):373-83. [PubMed: 23420871]

28. Mause SF. Platelet microparticles: reinforcing the hegemony of platelets in atherothrombosis. Thromb Haemost. 2013; 109(1):5-6. [PubMed: 23196668]

29. Heijnen HF, et al. Activated platelets release two types of membrane vesicles: microvesicles by surface shedding and exosomes derived from exocytosis of multivesicular bodies and alphagranules. Blood. 1999; 94(11):3791-9. [PubMed: 10572093]

30. von Bruhl ML, et al. Monocytes, neutrophils, and platelets cooperate to initiate and propagate venous thrombosis in mice in vivo. J Exp Med. 2012; 209(4):819-35. [PubMed: 22451716] 
31. Darbousset R, et al. Tissue factor-positive neutrophils bind to injured endothelial wall and initiate thrombus formation. Blood. 2012; 120(10):2133-43. [PubMed: 22837532]

32. Engelmann B, Massberg S. Thrombosis as an intravascular effector of innate immunity. Nat Rev Immunol. 2013; 13(1):34-45. [PubMed: 23222502]

33. Fuchs TA, et al. Extracellular DNA traps promote thrombosis. Proc Natl Acad Sci U S A. 2010; 107(36):15880-5. [PubMed: 20798043]

34. Demers M, et al. Cancers predispose neutrophils to release extracellular DNA traps that contribute to cancer-associated thrombosis. Proc NatlAcad Sci U SA. 2012; 109(32):13076-81.

35. Chen K, et al. Endocytosis of soluble immune complexes leads to their clearance by FcgammaRIIIB but induces neutrophil extracellular traps via FcgammaRIIA in vivo. Blood. 2012; 120(22):4421-31. [PubMed: 22955924]

36. Duerschmied D, et al. Platelet serotonin promotes the recruitment of neutrophils to sites of acute inflammation in mice. Blood. 2013; 121(6):1008-15. [PubMed: 23243271]

37. Martinod K, et al. Neutrophil histone modification by peptidylarginine deiminase 4 is critical for deep vein thrombosis in mice. Proc Natl Acad Sci U S A. 2013; 110(21):8674-9. [PubMed: 23650392]

38. Torisu T, et al. Autophagy regulates endothelial cell processing, maturation and secretion of von Willebrand factor. Nat Med. 2013; 19(10):1281-7. [PubMed: 24056772]

39. Li Z, et al. A stimulatory role for cGMP-dependent protein kinase in platelet activation. Cell. 2003; 112(1):77-86. [PubMed: 12526795]

40. Zhang G, et al. Biphasic roles for soluble guanylyl cyclase (sGC) in platelet activation. Blood. 2011; 118(13):3670-9. [PubMed: 21803853]

41. Gambaryan S, Friebe A, Walter U. Does the NO/sGC/cGMP/PKG pathway play a stimulatory role in platelets? Blood. 2012; 119(22):5335-6. author reply 5336-7. [PubMed: 22653960]

42. Tsikas D, et al. Extra-platelet $\mathrm{NO}$ and $\mathrm{NO}(+)$-containing drugs are potent inhibitors of platelet aggregation in humans by cGMP-dependent and cGMP-independent mechanisms. Blood. 2012; 119(22):5337-9. author reply 5339. [PubMed: 22653961]

43. Sylman JL, et al. Transport limitations of nitric oxide inhibition of platelet aggregation under flow. Ann Biomed Eng. 2013; 41(10):2193-205. [PubMed: 23563992]

44. Schulz C, Massberg S. Platelets in atherosclerosis and thrombosis. Handb Exp Pharmacol. 2012; 210:111-33.

45. Lam WA, et al. Mechanics and contraction dynamics of single platelets and implications for clot stiffening. Nat Mater. 2011; 10(1):61-6. [PubMed: 21131961]

46. Kita A, et al. Microenvironmental geometry guides platelet adhesion and spreading: a quantitative analysis at the single cell level. PLoS ONE. 2011; 6(10):e26437. [PubMed: 22028878]

47. Cines DB, et al. Clot contraction: compression of erythrocytes into tightly packed polyhedra and redistribution of platelets and fibrin. Blood. 2014; 123(10):1596-603. [PubMed: 24335500]

48. Westein E, et al. Atherosclerotic geometries exacerbate pathological thrombus formation poststenosis in a von Willebrand factor-dependent manner. Proc Natl Acad Sci U S A. 2013; 110(4):1357-62. [PubMed: 23288905]

49. Schumacher D, et al. Platelet-derived nucleotides promote tumor-cell transendothelial migration and metastasis via P2Y2 receptor. Cancer Cell. 2013; 24(1):130-7. [PubMed: 23810565]

50. Macaulay IC, et al. Platelet genomics and proteomics in human health and disease. J Clin Invest. 2005; 115(12):3370-7. [PubMed: 16322782]

51. Mason KD, et al. Programmed anuclear cell death delimits platelet life span. Cell. 2007; 128(6): 1173-86. [PubMed: 17382885]

52. Gieger C, et al. New gene functions in megakaryopoiesis and platelet formation. Nature. 2011; 480(7376):201-8. [PubMed: 22139419]

53. Jones CI, et al. A functional genomics approach reveals novel quantitative trait loci associated with platelet signaling pathways. Blood. 2009; 114(7):1405-16. [PubMed: 19429868]

54. Rowley JW, et al. Genome-wide RNA-seq analysis of human and mouse platelet transcriptomes. Blood. 2011; 118(14):e101-11. [PubMed: 21596849] 
55. Burkhart JM, et al. The first comprehensive and quantitative analysis of human platelet protein composition allows the comparative analysis of structural and functional pathways. Blood. 2012; 120(15):e73-e82. [PubMed: 22869793]

56. Dittrich M, et al. Platelet protein interactions: map, signaling components, and phosphorylation groundstate. Arterioscler Thromb Vasc Biol. 2008; 28(7):1326-31. [PubMed: 18451328]

57. Coppinger JA, et al. Characterization of the proteins released from activated platelets leads to localization of novel platelet proteins in human atherosclerotic lesions. Blood. 2004; 103(6):2096104. [PubMed: 14630798]

58. Pena E, et al. Proteomic signature of thrombin-activated platelets after in vivo nitric oxide-donor treatment: coordinated inhibition of signaling (phosphatidylinositol 3-kinase-gamma, 14-3-3zeta, and growth factor receptor-bound protein 2) and cytoskeleton protein translocation. Arterioscler Thromb Vasc Biol. 2011; 31(11):2560-9. [PubMed: 21836071]

59. Dittrich M, et al. Understanding platelets. Lessons from proteomics, genomics and promises from network analysis. Thromb Haemost. 2005; 94(5):916-25. [PubMed: 16363231]

60. Boyanova D, et al. PlateletWeb: a systems biologic analysis of signaling networks in human platelets. Blood. 2012; 119(3):e22-e34. [PubMed: 22123846]

61. Dittrich M, et al. Characterization of a novel interaction between vasodilator-stimulated phosphoprotein and Abelson interactor 1 in human platelets: a concerted computational and experimental approach. Arterioscler Thromb Vasc Biol. 2010; 30(4):843-50. [PubMed: 20110575]

62. Jupe $S$, et al. Reactome-a curated knowledgebase of biological pathways: megakaryocytes and platelets. J Thromb Haemost. 2012; 10(11):2399-402. [PubMed: 22985186]

63. $\mathrm{Xu} \mathrm{Z}$, et al. Computational approaches to studying thrombus development. Arterioscler Thromb Vasc Biol. 2011; 31(3):500-5. [PubMed: 21325672]

64. Flamm MH, et al. Multiscale prediction of patient-specific platelet function under flow. Blood. 2012; 120(1):190-8. [PubMed: 22517902]

65. Flamm MH, Diamond SL. Multiscale systems biology and physics of thrombosis under flow. Ann Biomed Eng. 2012; 40(11):2355-64. [PubMed: 22460075]

66. Stalker TJ, et al. Hierarchical organization in the hemostatic response and its relationship to the platelet-signaling network. Blood. 2013; 121(10):1875-85. [PubMed: 23303817]

67. Skorczewski T, Erickson LC, Fogelson AL. Platelet motion near a vessel wall or thrombus surface in two-dimensional whole blood simulations. Biophys J. 2013; 104(8):1764-72. [PubMed: 23601323]

68. Voronov RS, et al. Simulation of intrathrombus fluid and solute transport using in vivo clot structures with single platelet resolution. Ann Biomed Eng. 2013; 41(6):1297-307. [PubMed: 23423707]

69. Kim OV, et al. Fibrin networks regulate protein transport during thrombus development. PLoS Comput Biol. 2013; 9(6):e1003095. [PubMed: 23785270]

70. Leiderman K, Fogelson A. An overview of mathematical modeling of thrombus formation under flow. Thromb Res. 2014; 133(Suppl 1):S12-4. [PubMed: 24759131]

71. Pivkin IV, Richardson PD, Karniadakis G. Blood flow velocity effects and role of activation delay time on growth and form of platelet thrombi. Proc Natl Acad Sci U S A. 2006; 103(46):17164-9. [PubMed: 17085579]

72. Begent N, Born GV. Growth rate in vivo of platelet thrombi, produced by iontophoresis of ADP, as a function of mean blood flow velocity. Nature. 1970; 227(5261):926-30. [PubMed: 5448995]

73. Fogelson AL, Guy RD. Immersed-boundary-type models of intravascular platelet aggregation Comput Methods. Appl Mech Eng. 2008; 197:2087-104.

74. Mody NA, King MR. Platelet adhesive dynamics. Part I: characterization of platelet hydrodynamic collisions and wall effects. Biophys J. 2008; 95(5):2539-55. [PubMed: 18515387]

75. Huang PY, Hellums JD. Aggregation and disaggregation kinetics of human blood platelets: part III. The disaggregation under shear stress of platelet aggregates. Biophys J. 1993; 65(1):354-61. [PubMed: 8369443]

76. Huang PY, Hellums JD. Aggregation and disaggregation kinetics of human blood platelets: part II. Shear-induced platelet aggregation. Biophys J. 1993; 65(1):344-53. [PubMed: 8369442] 
77. Huang PY, Hellums JD. Aggregation and disaggregation kinetics of human blood platelets: part I. Development and validation of a population balance method. Biophys J. 1993; 65(1):334-43. [PubMed: 8369441]

78. Leiderman K, Fogelson AL. Grow with the flow: a spatial-temporal model of platelet deposition and blood coagulation under flow. Math Med Biol. 2011; 28(1):47-84. [PubMed: 20439306]

79. Kuharsky AL, Fogelson AL. Surface-mediated control of blood coagulation: the role of binding site densities and platelet deposition. Biophys J. 2001; 80(3):1050-74. [PubMed: 11222273]

80. Xu Z, et al. A multiscale model of thrombus development. J R Soc Interface. 2008; 5(24):705-22. [PubMed: 17925274]

81. Xu Z, et al. A multiscale model of venous thrombus formation with surface-mediated control of blood coagulation cascade. Biophys J. 2010; 98(9):1723-32. [PubMed: 20441735]

82. Xu Z, et al. Multiscale model of fibrin accumulation on the blood clot surface and platelet dynamics. Methods Cell Biol. 2012; 110:367-88. [PubMed: 22482956]

83. Xu Z, et al. Multiscale models of thrombogenesis. Wiley Interdiscip Rev Syst Biol Med. 2012; 4(3):237-46. [PubMed: 22246734]

84. Graner F, Glazier JA. Simulation of biological cell sorting using a two-dimensional extended Potts model. Phys Rev Lett. 1992; 69(13):2013-6. [PubMed: 10046374]

85. Stalker TJ, et al. A systems approach to hemostasis: 3 . Thrombus consolidation regulates intrathrombus solute transport and local thrombin activity. Blood. 2014; 124(11):1824-31. [PubMed: 24951426]

86. Wu Z, et al. Three-dimensional multi-scale model of deformable platelets adhesion to vessel wall in blood flow. Phil Trans R Soc A. 2014; 372(2021):1-23.

87. Diamond SL, et al. Systems biology of platelet-vessel wall interactions. Front Physiol. 2013; 4:229. [PubMed: 23986721] 
Leukocytes

NET:

\section{Platelets Blood Vessel geometry}


Blood flow direction shear and turbulences

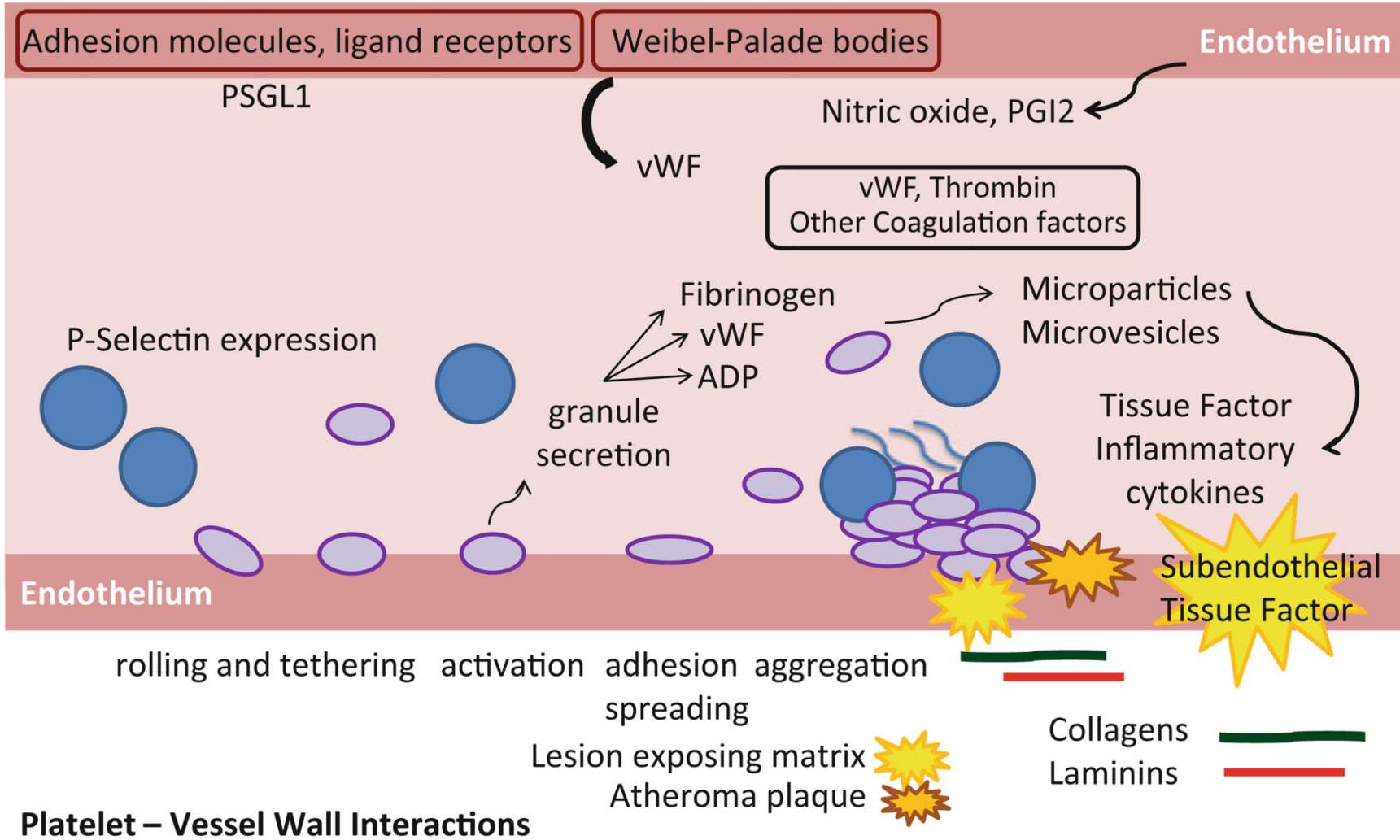

Fig. 5.1.

Platelets and vessel walls interact in multiple ways. Platelets and vessel wall cells express adhesion molecules, surface receptors, and release substances that initiate or regulate cascading thrombosis events. Matrix components of the vessel wall, when blood flow is exposed through a damaged endothelium, also elicit clotting reactions from blood cells. Moreover, blood cells and endothelial cells also produce microparticles and microvesicles that carry procoagulant activity 


\section{The complexity inside the platelets}

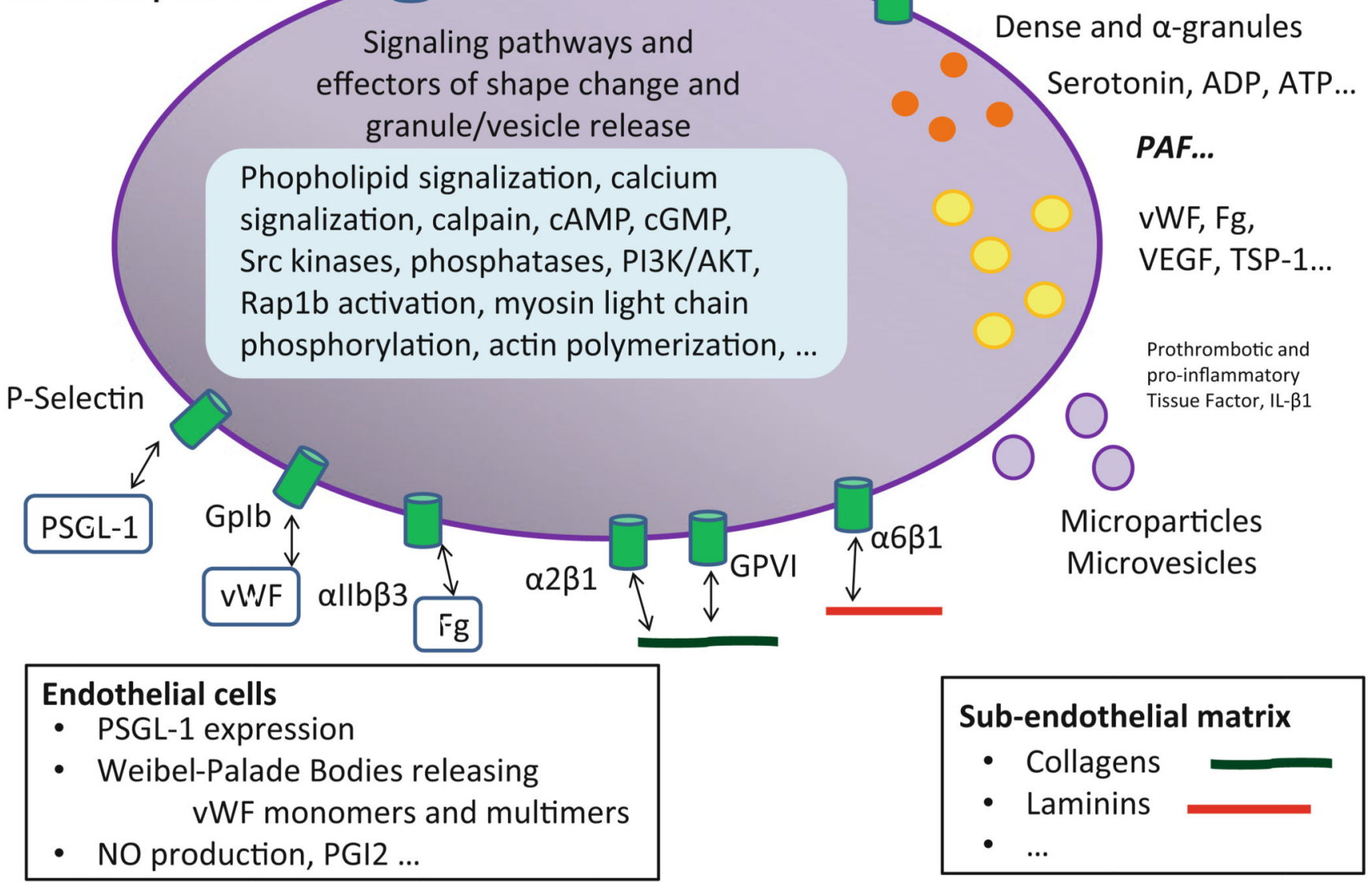

Fig. 5.2.

Platelets express a diversity of cell surface receptors and chemical substances that interact with the vessel wall components during hemostasis. Downstream signaling events take place that involve phospholipid signalization, $\mathrm{Ca}^{2+}$ flux, calpain activation, cAMP- and cGMPlevel modulation, cytoskeletal players and their modulators and diverse kinases. Many of these pathways intersect or interact with each other in a complex manner 


\section{Examples of focused scale approaches}

Larger scale and systemic approaches

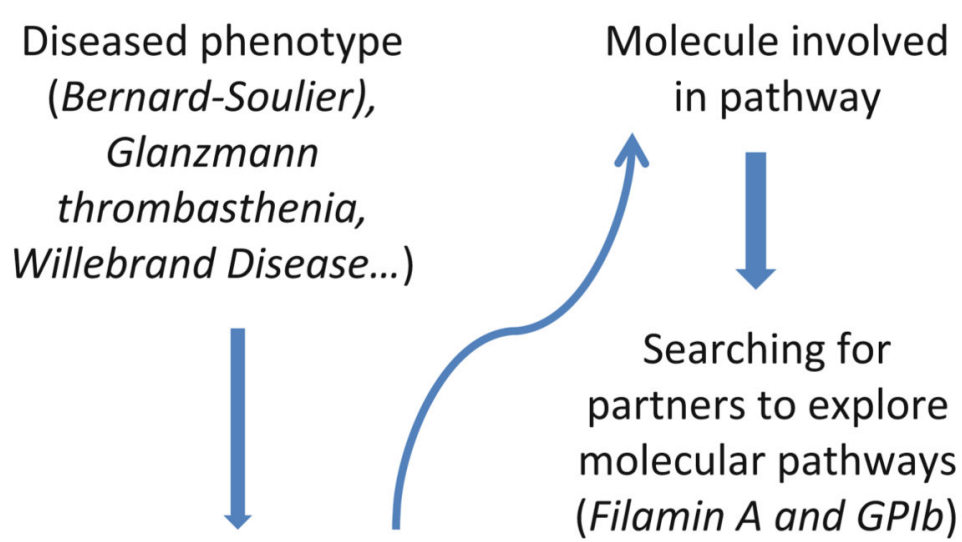

Molecule involved in pathway

Glanzmann

thrombasthenia,

Molecular actors and molecular pathways involved

(Gplb in Bernard - Soulier)
Inducing $\mathrm{KD} / \mathrm{KO} /$ Mutation of new molecule and observing behavior (Filamin A KO mice)

\section{Genomics Transcriptomics Proteomics}
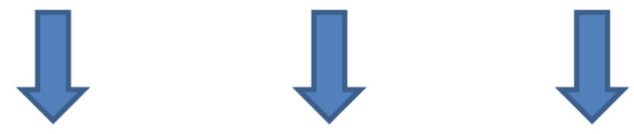

Network Analysis (PlateletWeb, Reactome)

Bioinformatics modeling

Fig. 5.3.

Systems approach to hemostasis control by platelet-blood vessel interaction. A highly informative approach is based on multi-scale analysis of human bleeding disorders at biochemical, molecular, genetic, and organismal levels 



8

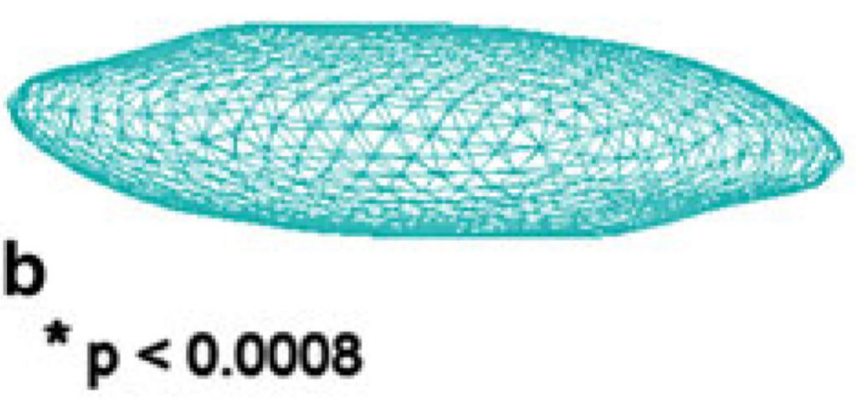

Fig. 5.4.

Simulated deformations of platelet structure during adhesion to the vessel wall for platelets stiffnesses of a $2.5 \mathrm{kPa}$ and $\mathbf{b} 25 \mathrm{kPa}$. $\mathbf{c}$ The effect of platelet membrane stiffness on the platelet pause time. (Originally published in Ref [86] Open Access: http:// rsta.royalsocietypublishing.org/content/372/2021/20130380.long) 